\title{
ON COVARIANCE GENERATING FUNCTIONS AND SPECTRAL DENSITIES OF PERIODICALLY CORRELATED AUTOREGRESSIVE PROCESSES
}

\author{
Z. SHISHEBOR, A. R. NEMATOLLAHI, AND A. R. SOLTANI \\ Received 11 May 2005; Revised 22 November 2005; Accepted 23 November 2005
}

Periodically correlated autoregressive nonstationary processes of finite order are considered. The corresponding Yule-Walker equations are applied to derive the generating functions of the covariance functions, what are called here the periodic covariance generating functions. We also provide closed formulas for the spectral densities by using the periodic covariance generating functions, which is a new technique in the spectral theory of periodically correlated processes.

Copyright (c) 2006 Z. Shishebor et al. This is an open access article distributed under the Creative Commons Attribution License, which permits unrestricted use, distribution, and reproduction in any medium, provided the original work is properly cited.

\section{Introduction}

In this work, we will consider periodically correlated autoregressive processes of finite order $p \geq 1(\operatorname{PCAR}(p))$. The aim is to develop a technique for analytic evaluation of the spectral densities. The work of Nematollahi and Soltani [6] treated the case of $p=1$. Their work exhibits the complexities in deriving closed forms for the spectral densities even for $p=1$. Moreover, it seems that their approach cannot even be extended for the case of $p>1$. Our approach, presented in this article, is new; it is based on employing the periodic covariance generating function (PCGF), which is the generating function of the covariance function. The $\operatorname{PCGF}$ for $\operatorname{PCAR}(p)$ has not yet been produced, to the best of our knowledge. Of course the significance of generating functions has been frequently realized in queueing, Markov chains, and most fields in engineering, but rarely in time series. We will derive the PCGF by using Yule-Walker equations for $\operatorname{PCAR}(p)$. Through some examples we will demonstrate the details of our method of deriving the PCGF.

The authors learned from a referee that Sakai [8] derived the formula for the spectral density matrix of periodic ARMA processes. The spectral part of this work is an alternative to other methods, but it is new in deriving the spectral density of $\operatorname{PCAR}(p)$ processes.

This paper is organized as follows. Preliminaries are given in Section 2. The PCGF for $\operatorname{PCAR}(p)$ is derived in Section 3. Closed forms for the spectral densities of $\operatorname{PCAR}(p)$ are

Hindawi Publishing Corporation

Journal of Applied Mathematics and Stochastic Analysis

Volume 2006, Article ID 94746, Pages 1-17

DOI 10.1155/JAMSA/2006/94746 
established in Section 4. Some examples are also provided, including a new derivation for the Nematollahi and Soltani [6] formula for the spectral density.

Concerning the literature on periodically correlated processes, periodically correlated AR or ARMA processes, we refer the reader to the works of Miamee [4], Pourahmadi and Salehi [7], Soltani and Parvardeh [10], and the references therein. For numerical computation of autocovariance function of periodically correlated ARMA processes, see Shao and Lund [9].

\section{Preliminaries}

A centered discrete-time second-order process $X=\left\{X_{t}, t \in \mathbb{Z}\right\}, \mathbb{Z}$ is the set of integers, is said to be $\operatorname{PCAR}(p)$ series if it is generated by the following model:

$$
X_{t}=c_{1}(t) X_{t-1}+c_{2}(t) X_{t-2}+\cdots+c_{p}(t) X_{t-p}+Z_{t}
$$

where $c_{i}(t), i=1, \ldots, p$, are periodic functions in $t$ with period $T$, and the process $\left\{Z_{t}\right\}$ is periodic white noise $\operatorname{PWN}\left(0, \sigma_{t}^{2}, T\right)$, which means (i) $E Z_{t}=0$, (ii) $\sigma_{t+T}^{2}=\sigma_{t}^{2}>0$, and (iii) $E Z_{t} Z_{s}=0$, for $t \neq s$. The smallest integer $T$ satisfying (2.1) is called the period. Note that if $T=1,(2.1)$ reduces to an ordinary AR model. In this work, we assume $T \geq 1$ and it stands for the period. The simplest way to justify the model in (2.1) is to apply the connection between PC and multivariate stationary processes, Gladyshev [2]. Indeed, let $\mathbf{Y}_{t}=\left(Y_{t}^{0}, Y_{t}^{1}, \ldots, Y_{t}^{T-1}\right)$, where $Y_{t}^{k}=X_{t T+k}$, and $\mathbf{W}_{t}=\left(W_{t}^{0}, W_{t}^{1}, \ldots, W_{t}^{T-1}\right)$, where $W_{t}^{k}=$ $Z_{t T+k}, t \in \mathbb{Z}, k=0, \ldots, T-1$. Then model (2.1) can be restated in the multivariate AR model

$$
\mathbf{Y}_{t}=\boldsymbol{\Phi}_{1} \mathbf{Y}_{t-1}+\cdots+\boldsymbol{\Phi}_{p^{*}} \mathbf{Y}_{t-p^{*}}+\mathbf{W}_{t}
$$

where $p^{*}=[p / T],[x]$ denotes the smallest integer greater than or equal to $x$. The $T \times T$ coefficients $\boldsymbol{\Phi}_{i}, i=1, \ldots, p^{*}$, are given in [9]. Let $\boldsymbol{\Phi}(z):=\mathbf{I}-\boldsymbol{\Phi}_{1} z-\cdots-\boldsymbol{\Phi}_{p^{*}} z^{p^{*}}$ be the $T$-variate AR polynomial associated with the process $\left\{\mathbf{Y}_{t}, t \in \mathbb{Z}\right\}$. It is well known that the process $\mathbf{Y}_{t}$ given by (2.2) uniquely exists and is stationary if $\operatorname{det}(\Phi(z)) \neq 0$, for all complex $|z| \leq 1[9]$.

Let $X=\left\{X_{t}, t \in \mathbb{Z}\right\}$ be a zero-mean $\operatorname{PCAR}(p)$, and let $R(t, s)=E X_{t} X_{s}$ denote the covariance function, then

$$
R(t, s)=R(t+T, s+T)
$$

for all $s, t$ in $\mathbb{Z}$. It follows from (2.3) that for each $k$, the function $R_{t}(k):=R(t+k, t)$ is periodic in $t$ with period $T$, that is,

$$
R_{t}(k)=R_{t+T}(k), \quad t, k \in \mathbb{Z} .
$$

It is plain to show that $R_{t}(k), t=0, \ldots, T-1, k \in \mathbb{Z}$, satisfy the following Yule-Walker-type, 
system of recursive relations:

$$
\begin{gathered}
\sum_{i=0}^{p} c_{i}(T-m) R_{T-(m+i)}(i)=-\sigma_{m}^{2}, \\
\sum_{i=0}^{p} c_{i}(T-m) R_{T-(m+i)}(i-k)=0, \quad m=0,1, \ldots, T-1, k \geq 1,
\end{gathered}
$$

where $\sigma_{m}^{2}=E Z_{T-m}^{2}$ and $c_{0}(s)=-1, s=1,2, \ldots, T$. Furthermore,

$$
R_{t}(l T+v)=R_{t+v}(-(l+1) T+(T-v)),
$$

for $l=0,1, \ldots$ and $t, v=0, \ldots, T-1$.

\section{A method to evaluate $R_{t}(k)$}

We apply the generating function method, see [3], for solving the system of recurrence equations given by (2.5). Let us first introduce the following notations and conventions:

$$
a_{i}(k):=R_{i}(-k), \quad i=0, \ldots, T-1, k=0,1, \ldots,
$$

$\lfloor u\rfloor$ and $\lfloor u\rfloor_{0}$ are the quotient and reminder in division of $u$ by $T$, that is, $u=\lfloor u\rfloor T+\lfloor u\rfloor_{0}$,

$$
\begin{gathered}
\mathcal{C}_{0}(s)=-1, \quad s=1,2, \ldots, T \\
A_{i+r T, j}(x)=A_{i, j}(x):=\sum_{k=0}^{j} a_{i}(k) x^{k}, \quad i=0,1, \ldots, T-1, r, j=0,1, \ldots,
\end{gathered}
$$

$A_{i+r T}(x)=A_{i}(x):=A_{i, \infty}(x),|x| \leq 1, x \in \mathbb{C}$, where $\mathbb{C}$ denotes the set of complex numbers,

$$
\begin{gathered}
e_{i+r T}(x)=e_{i}(x)=:-\sum_{j=0}^{p-1} c_{j}(i) x^{j} A_{i-j,(p-1)-j}(x), \quad i=0, \ldots, T-1, r \in \mathbb{Z}, \\
\mathbf{a}(x)=\left(\begin{array}{c}
A_{T}(x) \\
A_{T-1}(x) \\
\vdots \\
A_{1}(x)
\end{array}\right), \quad \mathbf{e}(x)=\left(\begin{array}{c}
e_{T}(x) \\
e_{T-1}(x) \\
\vdots \\
e_{1}(x)
\end{array}\right), \\
D_{i j}(x)= \begin{cases}M_{j-i}(x, T-i), & i=0,1, \ldots, T-1, j=0,1, \ldots, T-1, \\
N_{j-i}(x, T-i), & i=0,1, \ldots, T-1, j=\lfloor p\rfloor_{0}+1, \ldots, 2 T-2, \\
0, & \lfloor p\rfloor_{0}+1 \leq j-i \leq T-1,\end{cases}
\end{gathered}
$$


4 Covariance generating functions and spectral densities of PCAR

where $M_{l}(x, s)=-\sum_{u=0}^{\lfloor p\rfloor} c_{u T+l}(s) x^{u T+l}$, for $l=0,1, \ldots,\lfloor p\rfloor_{0}$, and

$$
\begin{aligned}
& N_{l}(x, s)= \begin{cases}-\sum_{u=0}^{\lfloor p\rfloor-1} c_{u T+l}(s) x^{u T+l}, & l=\lfloor p\rfloor_{0}+1, \ldots, T-1,\lfloor p\rfloor>0, \\
0, & \lfloor p\rfloor=0,\end{cases} \\
& \mathbf{D}(x)=\left[D_{i j}(x)\right], \quad i=0,1, \ldots, T-1, j=0,1, \ldots, 2 T-2 .
\end{aligned}
$$

Our aim is to specify the periodic covariance generating functions (PCGFs) $A_{i}(x), i=$ $0, \ldots, T-1$, then the coefficients $a_{i}(k)$, so $R_{i}(k)$, can be easily determined. For solving the system of equations in Theorems 3.2 and 3.9, as we will see in the following, we need to know the vector $\mathbf{e}(x)=\left(e_{T}(x), e_{T-1}(x), \ldots, e_{1}(x)\right)^{\prime}$.

Lemma 3.1. The vector $\mathbf{e}(x)$ is specified by the coefficients $\left\{a_{i}(k) ; k=0, \ldots, p-1, i=0, \ldots\right.$, $T-1\}$, which can be obtained from solving the following system of equations:

$$
\begin{gathered}
\sum_{i=0}^{p} c_{i}(T-m) a_{\lfloor T-(m+\lfloor i\rfloor T)\rfloor_{0}}(i)=-\sigma_{m}^{2}, \\
\sum_{i=0}^{k} c_{i}(T-m) a_{\lfloor T-(m+i)\rfloor_{0}}(k-i)+\sum_{i=k+1}^{p} c_{i}(T-m) a_{\left\lfloor T-(m+i)+\lfloor i-k\rfloor_{0}\right\rfloor_{0}}(i-k)=0,
\end{gathered}
$$

for $k=1, \ldots, p$ and $m=0, \ldots, T-1$.

Proof. It is clear that the vector $\mathbf{e}(x)$ is fully specified by $\left\{a_{i}(k) ; k=0, \ldots, p-1, i=\right.$ $0, \ldots, T-1\}$, which is the unique solution to the linear equations given above. By using the first equation given by $(2.5)$ and the fact that $R_{T-(m+i)}(i)=R_{T-m-\lfloor i] T}(-i)$ for $i \geq 0$, we will obtain the first system of equations given above. The second system of equation can be easily deduced by using the second equations given by $(2.5)$ and the fact that $R_{T-(m+i)}(i-k)=R_{T-(m+i)+\lfloor i-k\rfloor_{0}}(k-i)$ for $i>k$. The proof is complete.

Theorem 3.2. The periodic covariance generating functions $A_{i}(x), i=0, \ldots, T-1$, are given by

$$
\mathbf{B}(x) \mathbf{a}(x)=\mathbf{e}(x)
$$

where $\mathbf{B}(x)$ is the $T \times T$ matrix formed by first $T$ columns of

$$
\mathbf{S}(x)=\mathbf{D}(x) \mathbf{U}_{T-1,2 T-1} \mathbf{U}_{T-2,2 T-2} \mathbf{U}_{T-3,2 T-3} \cdots \mathbf{U}_{1, T+1},
$$

and each $\mathbf{U}_{j, r}$ is the matrix formed from a $(2 T-1) \times(2 T-1)$ identity matrix, $\mathbf{I}$, in this way that the $j$ th column of $\mathbf{U}_{j, r}$ is the sum of $r$ th and $j$ th columns of $\mathbf{I}$, and other columns of $\mathbf{U}_{j, r}$ are the same as those of $\mathbf{I}$. 
Proof. It easily follows from $(2.5)$ that for $m=0,1, \ldots, T-1$,

$$
\sum_{i=0}^{p} c_{i}(T-m) \sum_{k=0}^{\infty} a_{T-(m+i)}(k+p-i) x^{k+p}=0 .
$$

So the system of equations given above can be written as

$$
\sum_{i=0}^{p} c_{i}(T-m) x^{i} A_{T-i-m}(x)=-e_{T-m}(x), \quad m=0,1, \ldots, T-1
$$

Since $A_{i}(x), i=0,1, \ldots, T-1$, are periodic in $i$ with period $T$, the system of equations given above can also be written as follows:

$$
\mathbf{D}(x) \widetilde{\mathbf{a}}(x)=\mathbf{e}(x)
$$

where $\tilde{\mathbf{a}}(x)=\left(A_{T}(x), A_{T-1}(x), \ldots, A_{1}(x), A_{T}(x), \ldots, A_{2}(x)\right)^{\prime}$. To obtain (3.6) from (3.10), first form $\mathbf{S}(x)$ as in (3.7) and let $\mathbf{B}(x)$ be the matrix formed by first $T$ columns of $\mathbf{S}(x)$. Then it is easy to see that $A_{i}(x), i=0,1, \ldots, T-1$, satisfy the system of (3.6).

Remark 3.3. According to the causality assumption, the covariance functions are welldefined uniquely. Thus for each $x,|x| \leq 1$, the system of equations in (3.6) possesses a unique solution for $\mathbf{a}(x)$, giving that $\mathbf{B}(x)$ is invertible.

Example 3.4. Let $X$ be a causal PCAR(1) process with period $T=1$, with $c_{1}(t)=c$ and $\sigma_{t}^{2}=\sigma^{2}$. In this case, the matrix $\mathbf{B}(x)$ reduces to

$$
\mathbf{B}(x)=1-c x,
$$

so the model is causal if and only if $|c|<1$. Also, it is easily shown that $E X_{t}^{2}=\sigma^{2} /\left(1-c^{2}\right)$ and so

$$
A(x)=\frac{\sigma^{2}}{\left(1-c^{2}\right)(1-c x)},
$$

and hence

$$
R(k)=\frac{\sigma^{2} c^{|k|}}{\left(1-c^{2}\right)}
$$

for $k \in \mathbb{Z}$. This is in adaptation with the usual stationary autoregressive models of order one.

Example 3.5. Consider a causal PCAR(1) process with period $T, T \geq 2$. Here $c_{i}(t)=0$, $i=2, \ldots, p$. Let $c(t):=c_{1}(t)$. By using the method mentioned in Theorem 3.2, the elements of the matrices $\mathbf{D}(x)=\left[D_{i j}(x)\right]_{i=0, \ldots, T-1, j=0, \ldots, 2 T-2}, \mathbf{B}(x)=\left[B_{i j}(x)\right]_{i, j=0, \ldots, T-1}$, 
6 Covariance generating functions and spectral densities of PCAR

$\mathbf{B}^{-1}(x)=\left[B_{i j}^{\#}(x)\right]_{i, j=0, \ldots, T-1}$, and $\mathbf{e}(x)=\left[e_{j}(x)\right]_{j=0, \ldots, T-1}$ are given by

$$
\begin{aligned}
& D_{i j}(x)= \begin{cases}1, & i=j, \\
-c(T-j+1) x, & i=j-1 \\
0, & \text { otherwise }\end{cases} \\
& B_{i j}(x)= \begin{cases}1, & i=j, \\
-c(T-j+1) x, & i=j-1, \\
-c(1), & i=T-1, j=0, \\
0, & \text { otherwise, }\end{cases} \\
& B_{i j}^{\#}(x)=\frac{1}{1-\left(\prod_{z=1}^{T} c(z)\right) x^{T}} \begin{cases}1, & i=j, \\
\prod_{z=i}^{j-1} c(T-z) x^{j-i}, & j>i, \\
\left(\prod_{z=1}^{T-i} c(z) \prod_{z=0}^{j-1} c(T-z)\right) x^{T-(i-j)}, & i>j,\end{cases}
\end{aligned}
$$

$e_{j}(x)=R_{j}(0), j=0, \ldots, T-1$, respectively. By applying Theorem 3.2 and after some algebraic manipulations, we obtain

$$
R_{t}(-l T+v)=[c(0) c(1) \cdots c(T-1)]^{l-1}[c(t) c(t-1) \cdots(t-T+1+v)] R_{t+v}(0)
$$

for $t, v=0,1, \ldots, T-1, l=0,1, \ldots$ Now if we take $g(t-1):=c(t)$, then

$$
\begin{aligned}
R_{t}(-l T+v) & =[g(0) g(1) \cdots g(T-1)]^{l-1}[g(t-1) g(t-2) \cdots g(t-T+v)] R_{t+v}(0), \\
& =[\tilde{g}(T-1)]^{l-1} \tilde{g}(T+t-1)[\tilde{g}(t+v-1)]^{-1} R_{t+v}(0),
\end{aligned}
$$

where $\tilde{g}(n)=\prod_{j=0}^{n} g(j)$, which is the formula given in [6]. In the above equation, we have only $T$ unknown values $R_{j}(0), j=0, \ldots, T-1$, which are determined by Lemma 3.1 as follows:

$$
\begin{aligned}
& a_{\lfloor T-m\rfloor_{0}}(0)-c_{1}(T-m) a_{\lfloor T-m\rfloor_{0}}(1)=\sigma_{m}^{2}, \\
& a_{\lfloor T-m\rfloor_{0}}(1)-c_{1}(T-m) a_{\lfloor T-m-1\rfloor_{0}}(0)=0,
\end{aligned}
$$


for $m=0,1, \ldots, T-1$. After solving these equations, we conclude that

$$
\begin{gathered}
R_{T-i}(0)=a_{T-i}(0)=\frac{\sigma_{i}^{2}+c_{1}^{2}(T-i) \sigma_{i+1}^{2}+\cdots+c_{1}^{2}(T-i) \cdots c_{1}^{2}(-i+2) \sigma_{i+T-1}^{2}}{1-c_{1}^{2}(T) \cdots c_{1}^{2}(1)}, \\
a_{T-i}(1)=\frac{c_{1}^{2}(T-i) \sigma_{i+1}^{2}+\cdots+c_{1}^{2}(T-i) \cdots c_{1}^{2}(-i+1) \sigma_{T+i}^{2}}{c_{1}(T-i)\left[1-c_{1}^{2}(T) \cdots c_{1}^{2}(1)\right]},
\end{gathered}
$$

for $i=0, \ldots, T-1$.

Example 3.6. Let $X$ be a causal PCAR(2) process with period $T, T \geq 3$. In this case, the equations in Lemma 3.1 reduces to

$$
\begin{gathered}
a_{\lfloor T-m\rfloor_{0}}(0)-c_{1}(T-m) a_{\lfloor T-m\rfloor_{0}}(1)-c_{2}(T-m) a_{\lfloor T-m\rfloor_{0}}(2)=\sigma_{m}^{2}, \\
a_{\lfloor T-m\rfloor_{0}}(1)-c_{1}(T-m) a_{\lfloor T-m-1\rfloor_{0}}(0)-c_{2}(T-m) a_{\lfloor T-m-1\rfloor_{0}}(1)=0, \\
a_{\lfloor T-m\rfloor_{0}}(2)-c_{1}(T-m) a_{\lfloor T-m-1\rfloor_{0}}(1)-c_{2}(T-m) a_{\lfloor T-m-2\rfloor_{0}}(0)=0,
\end{gathered}
$$

for $m=0,1, \ldots, T-1$. From (3.8) we have

$$
\sum_{i=0}^{2} c_{i}(T-m) \sum_{k=0}^{\infty} a_{T-(m+i)}(k+2-i) x^{k+2}=0, \quad m=0,1, \ldots, T-1 .
$$

So

$$
\begin{gathered}
A_{T}(x)-c_{1}(T) x A_{T-1}(x)-c_{2}(T) x^{2} A_{T-2}(x)=e_{T}(x), \\
A_{T-1}(x)-c_{1}(T-1) x A_{T-2}(x)-c_{2}(T-1) x^{2} A_{T-3}(x)=e_{T-1}(x), \\
\vdots \\
A_{1}(x)-c_{1}(1) x A_{0}(x)-c_{2}(1) x^{2} A_{-1}(x)=e_{1}(x),
\end{gathered}
$$

and the elements of the matrices $\mathbf{D}(x)$ and $\mathbf{B}(x)$ are given by

$$
\begin{gathered}
D_{i j}(x)= \begin{cases}1, & i=j, \\
-c_{1}(T-j+1) x, & i=j-1, \\
-c_{2}(T-j+2) x^{2}, & 0 i=j-2, \\
0, & \text { otherwise, }\end{cases} \\
\qquad B_{i j}(x)= \begin{cases}1, & i=j, \ldots, T-1, j=0, \ldots, 2 T-2, \\
-c_{1}(T-j+1) x, & i=j-1, \\
-c_{2}(T-j+2) x^{2}, & i=j-2, \\
-c_{2}(2) x^{2}, & j=0, i=T-2, \\
-c_{1}(1) x, & j=0, i=T-1, \\
-c_{2}(1) x^{2}, & j=1, i=T-1, \\
0, & \text { otherwise. }\end{cases}
\end{gathered}
$$


8 Covariance generating functions and spectral densities of PCAR

Now (3.6) can be solved for $\mathbf{a}(x)$ by using a mathematical software.

Example 3.7. Let $X$ be a causal PCAR(2) with period $T=1$ (stationary case), with $c_{1}(t)=$ $c_{1}, c_{2}(t)=c_{2}$. In this case, the formula (3.6) takes the simple form

$$
A(x):=A_{1}(x)=\frac{a(0)+a(1) x-c_{1} x a(0)}{1-c_{1} x-c_{2} x^{2}}
$$

The covariance generating function for $A R(2)$ is defined in [1, Page 103] as

$$
\begin{aligned}
G(x) & =\sum_{k=-\infty}^{\infty} a(k) x^{k}, \quad(a(k)=a(-k)) \\
& =\frac{\sigma^{2}}{\left(1-c_{1} x-c_{2} x^{2}\right)\left(1-c_{1} / x-c_{2} / x^{2}\right)} .
\end{aligned}
$$

Therefore, in our settings, $G(x)$ and $A(x)$ are related through

$$
G(x)=A(x)+A\left(\frac{1}{x}\right)-a(0)
$$

where $A(x)=\sum_{k=0}^{\infty} a(k) x^{k}$, and its closed form is given by (3.23). It can be readily verified that $A(x)$ in (3.23) and $G(x)$ in (3.24) satisfy (3.25) in view of the following boundary conditions:

$$
\begin{aligned}
& a(0)-c_{1} a(1)-c_{2} a(2)=\sigma^{2}, \\
& a(1)-c_{1} a(0)-c_{2} a(1)=0, \\
& a(2)-c_{1} a(1)-c_{2} a(0)=0 .
\end{aligned}
$$

In the following, we consider the simple case $T=p=2$, which will clarify the proof of Theorem 3.2.

Example 3.8. Let $X$ be a PCAR(2) process with period $T=2$, then from Lemma 3.1, for $m=0,1$, we have

$$
\begin{aligned}
& a_{\lfloor 2-m\rfloor_{0}}(0)-c_{1}(2-m) a_{\lfloor 2-m\rfloor_{0}}(1)-c_{2}(2-m) a_{\lfloor 2-m\rfloor_{0}}(2)=\sigma_{m}^{2}, \\
& a_{\lfloor 2-m\rfloor_{0}}(1)-c_{1}(2-m) a_{\lfloor 1-m\rfloor_{0}}(0)-c_{2}(2-m) a_{\lfloor 1-m\rfloor_{0}}(1)=0, \\
& a_{\lfloor 2-m\rfloor_{0}}(2)-c_{1}(2-m) a_{\lfloor 1-m\rfloor_{0}}(1)-c_{2}(2-m) a_{\lfloor 2-m\rfloor_{0}}(0)=0 .
\end{aligned}
$$


It follows that

$$
\begin{aligned}
& a_{0}(0)=\frac{1}{H}\left[\left(-c_{2}(1) c_{2}(2)+c_{2}^{3}(1) c_{2}(2)-c_{2}^{2}(1)+1-2 c_{1}(1) c_{1}(2) c_{2}(1)\right) \sigma_{0}^{2}\right. \\
& \left.+\left(c_{2}(1) c_{2}(2) c_{1}^{2}(2)+c_{1}^{2}(2)\right) \sigma_{1}^{2}\right] \\
& a_{0}(1)=-\frac{1}{H}\left[\left(-c_{1}^{2}(1) c_{1}(2)-c_{1}(1) c_{2}(2)+c_{2}(2) c_{1}(1) c_{2}^{2}(1)\right) \sigma_{0}^{2}\right. \\
& \left.+\left(c_{1}(2) c_{2}^{2}(2)+c_{2}(2) c_{1}(1) c_{1}^{2}(2)-c_{1}(2)\right) \sigma_{1}^{2}\right], \\
& a_{0}(2)=\frac{1}{H}\left[\left(-c_{1}^{2}(1) c_{2}(1) c_{1}(2)+c_{1}(2) c_{1}(1)-c_{1}(2) c_{2}^{2}(1) c_{1}(1)-c_{2}(1) c_{2}^{2}(2)\right.\right. \\
& \left.+c_{2}^{3}(1) c_{2}^{2}(2)-c_{2}(2) c_{2}^{2}(1)+c_{2}(2)-2 c_{1}(1) c_{1}(2) c_{2}(1) c_{2}(2)\right) \sigma_{0}^{2} \\
& \left.+\left(c_{2}(1) c_{1}^{2}(2)+c_{1}(1) c_{1}^{3}(2)+c_{2}(2) c_{1}^{2}(2)\right) \sigma_{1}^{2}\right], \\
& a_{1}(0)=-\frac{1}{H}\left[\left(-c_{1}^{2}(1)-c_{1}^{2}(1) c_{2}(1) c_{2}(2)\right) \sigma_{0}^{2}\right. \\
& \left.+\left(c_{2}(1) c_{2}(2)-c_{2}(1) c_{2}^{3}(2)+c_{2}^{2}(2)+2 c_{1}(2) c_{2}(2) c_{1}(1)-1\right) \sigma_{1}^{2}\right], \\
& a_{1}(1)=\frac{1}{H}\left[\left(-c_{1}(2) c_{1}^{2}(1) c_{2}(1)+c_{1}(1)-c_{2}^{2}(1) c_{1}(1)\right) \sigma_{0}^{2}\right. \\
& \left.+\left(c_{2}(1) c_{1}(2)-c_{2}^{2}(2) c_{2}(1) c_{1}(2)+c_{1}(1) c_{1}^{2}(2)\right) \sigma_{1}^{2}\right], \\
& a_{1}(2)=-\frac{1}{H}\left[\left(-c_{1}^{3}(1) c_{1}(2)-c_{2}(1) c_{1}^{2}(1)-c_{2}(2) c_{1}^{2}(1)\right) \sigma_{0}^{2}\right. \\
& +\left(c_{2}^{2}(1) c_{2}(2)-c_{2}(1)-c_{2}^{2}(1) c_{2}^{3}(2)+c_{2}^{2}(2) c_{2}(1)+c_{2}(2) c_{1}^{2}(1) c_{1}^{2}(2)\right. \\
& \left.\left.+2 c_{1}(1) c_{1}(2) c_{2}(1) c_{2}(2)-c_{1}(1) c_{1}(2)+c_{1}(1) c_{2}^{2}(2) c_{1}(2)\right) \sigma_{1}^{2}\right],
\end{aligned}
$$

where

$$
\begin{aligned}
H= & \left(c_{2}(1) c_{2}(2)-1\right)\left[c_{1}(1) c_{1}(2)-1+c_{2}(1)+c_{2}(2)-c_{2}(1) c_{2}(2)\right] \\
& \times\left[c_{1}(1) c_{1}(2)+1+c_{2}(1)+c_{2}(2)+c_{2}(1) c_{2}(2)\right] .
\end{aligned}
$$

The system of (3.8) reduces to

$$
\begin{aligned}
& \sum_{k=0}^{\infty} a_{2}(k+2) x^{k+2}-c_{1}(2) \sum_{k=0}^{\infty} a_{1}(k+1) x^{k+2}-c_{2}(2) \sum_{k=0}^{\infty} a_{0}(k) x^{k+2}=0, \\
& \sum_{k=0}^{\infty} a_{1}(k+2) x^{k+2}-c_{1}(1) \sum_{k=0}^{\infty} a_{0}(k+1) x^{k+2}-c_{2}(1) \sum_{k=0}^{\infty} a_{-1}(k) x^{k+2}=0 .
\end{aligned}
$$


10 Covariance generating functions and spectral densities of PCAR

which can be written as

$$
\begin{aligned}
& A_{2}(x)-c_{1}(2) x A_{1}(x)-c_{2}(2) x^{2} A_{0}(x)=a_{0}(0)+a_{0}(1) x-c_{1}(2) a_{1}(0) x, \\
& A_{1}(x)-c_{1}(1) x A_{0}(x)-c_{2}(1) x^{2} A_{-1}(x)=a_{1}(0)+a_{1}(1) x-c_{1}(1) a_{0}(0) x .
\end{aligned}
$$

Thus

$$
\begin{gathered}
\mathbf{D}(x)=\left(\begin{array}{ccc}
1-c_{2}(2) x^{2} & -c_{1}(2) x & 0 \\
0 & 1-c_{2}(1) x^{2} & -c_{1}(1) x
\end{array}\right), \\
\widetilde{\mathbf{a}}(x)=\left(\begin{array}{l}
A_{2}(x) \\
A_{1}(x) \\
A_{2}(x)
\end{array}\right), \quad \mathbf{e}(x)=\left(\begin{array}{l}
a_{0}(0)+a_{0}(1) x-c_{1}(2) a_{1}(0) x \\
a_{1}(0)+a_{1}(1) x-c_{1}(1) a_{0}(0) x
\end{array}\right) .
\end{gathered}
$$

Now

$$
\mathbf{S}(x)=\mathbf{D}(x) \mathbf{U}_{1,3}=\left(\begin{array}{ccc}
1-c_{2}(2) x^{2} & -c_{1}(2) x & 0 \\
-c_{1}(1) x & 1-c_{2}(1) x^{2} & -c_{1}(1) x
\end{array}\right)
$$

giving that

$$
\mathbf{B}(x)=\left(\begin{array}{cc}
1-c_{2}(2) x^{2} & -c_{1}(2) x \\
-c_{1}(1) x & 1-c_{2}(1) x^{2}
\end{array}\right)
$$

Now by solving $\mathbf{B}(x) \mathbf{a}(x)=\mathbf{e}(x)$, where $\mathbf{a}(x)=\left(\begin{array}{c}A_{2}(x) \\ A_{1}(x)\end{array}\right)$, we obtain that

$$
A_{1}(x)=\frac{\mathcal{N}_{1}(x)}{\mathscr{D}(x)}, \quad A_{2}(x)=\frac{\mathcal{N}_{2}(x)}{\mathscr{D}(x)},
$$

where

$$
\begin{aligned}
\mathcal{N}_{1}(x)= & c_{2}(2)\left(c_{1}(1) a_{0}(0)-a_{1}(1)\right) x^{3} \\
& +\left(c_{1}(1) a_{0}(1)-c_{1}(1) c_{1}(2) a_{1}(0)-c_{2}(2) a_{1}(0)\right) x^{2}+a_{1}(1) x+a_{1}(0), \\
\mathcal{N}_{2}(x)= & c_{2}(1)\left(c_{1}(2) a_{1}(0)-a_{0}(1)\right) x^{3} \\
& +\left(c_{1}(2) a_{1}(1)-c_{1}(2) c_{1}(1) a_{0}(0)-c_{2}(1) a_{0}(0)\right) x^{2}+a_{0}(1) x+a_{0}(0), \\
\mathscr{D}(x)= & c_{2}(1) c_{2}(2) x^{4}-\left(c_{1}(1) c_{1}(2)+c_{2}(1)+c_{2}(2)\right) x^{2}+1
\end{aligned}
$$

where the $a_{0}(0), a_{0}(1), a_{1}(0)$, and $a_{1}(1)$ are given by (3.28). 
In the following, we will examine the structure of the PCGF $\left\{A_{l}(x), l=0, \ldots, T-1\right\}$ in order to establish its relation with the spectral density of the process. Interestingly,

$$
A_{l}(x)=\sum_{j=0}^{T-1} A_{l}^{j}(x), \quad l=0, \ldots, T-1
$$

where

$$
A_{l}^{j}(x)=\sum_{k=0}^{\infty} R_{l}(-k T-j) x^{k T+j}, \quad j, l=0,1, \ldots, T-1 .
$$

Let us call $A_{l}^{j}(x)$ the " $j$ th partition" of $A_{l}(x)$. For $T=2$, these partitions can be specified from the corresponding PCGF $A_{l}(x)$ through

$$
A_{l}^{0}(x)=\frac{A_{l}(x)+A_{l}(-x)}{2}, \quad A_{l}^{1}(x)=\frac{A_{l}(x)-A_{l}(-x)}{2} .
$$

It is not clear if this is the case for $T>2$. Nevertheless, the following theorem indicates that these partitions are solutions to $T$ linear systems each consisting of $T$ linear equations.

Let us recall that based on our notation every $u$ uniquely can be written as $u=\lfloor u\rfloor T+$ $\lfloor u\rfloor_{0}$.

Theorem 3.9. The " $j$ th partition" of $A_{l}(x)$ is given by

$$
\sum_{l=0}^{p} c_{l}(T-m) x^{l} A_{T-(m+l)}^{\lfloor j+p-l\rfloor_{0}}(x)=e_{j, T-m}(x), \quad m=0,1, \ldots, T-1, j=\lfloor T-m+r\rfloor_{0},
$$

for any $r=0,1, \ldots, T-1$, where

$$
e_{j, T-m}(x)=-\sum_{l=0}^{p} c_{l}(T-m) x^{l} \sum_{k=0}^{\lfloor j+p-l\rfloor-1} a_{T-(m+l)}\left(k T+\lfloor j+p-l\rfloor_{0}\right) x^{k T+\lfloor j+p-l\rfloor_{0}} .
$$

Proof. . Similar to (3.8), we can write

$$
\sum_{l=0}^{p} \sum_{k=0}^{\infty} c_{l}(T-m) a_{T-(m+l)}\left(k T+\lfloor j+p-l\rfloor T+\lfloor j+p-l\rfloor_{0}\right) x^{k T+\lfloor j+p\rfloor T+\lfloor j+p\rfloor_{0}}=0,
$$

for $m, j=0, \ldots, T-1$, which leads to (3.40) after some algebraic simplifications. 
Remark 3.10. Note that clearly the argument $k T+\lfloor j+p-l\rfloor_{0}$ is nonnegative and takes its maximum whenever $k=\lfloor j+p-l\rfloor-1$. In this case, we have $k T+\lfloor j+p-l\rfloor_{0}=(\lfloor j+$ $p-l\rfloor-1) T+\lfloor j+p-l\rfloor_{0}=j+p-l-T$, which is at most $p-1$. So all $e_{j, T-m}(x)$ in (3.41) are completely determined using Lemma 3.1.

The classification in equation (3.40) is crucial in finding the partitions. Indeed, since $A_{\left\lfloor s+k^{\prime} T\right\rfloor}^{l+k T}(x)=A_{\lfloor s\rfloor}^{l}(x)$, for all $k, k^{\prime} \in \mathbb{Z}$, the classification in (3.40) takes places, which enables one to solve each system separately for any $r=0,1, \ldots, T-1$, as in Theorem 3.2.

\section{A characterization for the spectral density matrix of a $\operatorname{PCAR}(p)$ process}

The spectral density of a PC process was introduced in [2], if it exists, it is a Hermitian nonnegative definite $T \times T$ matrix of complex functions on $[0,2 \pi)$,

$$
\mathbf{f}(\lambda)=\left[f_{j k}(\lambda)\right]_{j, k=0,1, \ldots, T-1},
$$

for which

$$
\begin{gathered}
R_{t}(\tau)=\sum_{k=0}^{T-1} B_{k}(\tau) \exp \left(\frac{2 \pi i k t}{T}\right), \\
B_{k}(\tau)=\int_{0}^{2 \pi} e^{i \tau \lambda} f_{k}(\lambda) d(\lambda),
\end{gathered}
$$

where $f_{k}(\lambda)$ and $f_{j k}(\lambda), j, k=0,1, \ldots, T-1$, are related through

$$
f_{j k}(\lambda)=\frac{1}{T} f_{k-j}\left(\frac{\lambda-2 \pi j}{T}\right), \quad j, k=0,1, \ldots, T-1,0 \leq \lambda<2 \pi .
$$

In this section, we characterize $\mathbf{f}(\lambda)$ of a $\operatorname{PCAR}(p)$ process. As we mentioned in Section 2, corresponding to every PC process $X_{t}, t \in \mathbb{Z}$, with period $T$, the $T$-dimensional random sequence $\mathbf{Y}_{t}=\left(Y_{t}^{0}, Y_{t}^{1}, \ldots, Y_{t}^{T-1}\right)$, where $Y_{t}^{k}=X_{t T+k}, t \in \mathbb{Z}, k=0, \ldots, T-1$, is stationary in the wide sense, and from the causality, the spectral distribution matrix of the process $\mathbf{Y}_{t}$ has a uniformly continuous spectral density matrix $\mathbf{h}(\lambda)=\left[h_{j k}(\lambda)\right]_{j, k=0,1, \ldots, T-1}$. Moreover,

$$
h_{j k}(\lambda)=\frac{1}{2 \pi} \sum_{\tau=-\infty}^{\infty} Q_{j k}(\tau) e^{-i \lambda \tau}, \quad 0 \leq \lambda<2 \pi,
$$

where $\mathbf{Q}(\tau)=\left[Q_{j k}(\tau)\right]_{j, k=0,1, \ldots, T-1}$ is the covariance matrix of $\mathbf{Y}_{t}$.

Lemma 4.1. The spectral density matrix $\mathbf{h}(\lambda)=\left[h_{j k}(\lambda)\right]_{j, k=0,1, \ldots, T-1}$ of the T-variate stationary process $\mathbf{Y}_{t}$ is specified by

$$
h_{j k}(\lambda)=\frac{1}{2 \pi} \exp \left(-\frac{i \lambda(k-j)}{T}\right)\left[A_{k}^{\langle k-j\rangle-J_{\langle k-j\rangle}}\left(e^{i \lambda / T}\right)+A_{j}^{\langle j-k\rangle}\left(e^{-i \lambda / T}\right)\right]
$$


for $j, k=0,1, \ldots, T-1,0 \leq \lambda<2 \pi$, where

$$
\langle s\rangle=\left\{\begin{array}{ll}
s, & s>0, \\
s+T, & s \leq 0,
\end{array} \quad J_{u}= \begin{cases}T, & u=T \\
0, & u \neq T\end{cases}\right.
$$

and $A_{l}^{j}$ are the partitions given in Theorem 3.9.

Proof. Note that for $j, k=0,1, \ldots, T-1$,

$$
\begin{aligned}
Q_{j k}(\tau) & =E Y_{t+\tau}^{j} Y_{t}^{k}=E X_{(t+\tau) T+j} X_{t T+k} \\
& =E X_{\tau T+j} X_{k}=E X_{\tau T+j-k+k} X_{k}=R_{k}(\tau T+j-k) .
\end{aligned}
$$

Consequently, for $0 \leq \lambda<2 \pi$ and $j \leq k$, we obtain that

$$
\begin{aligned}
h_{j k}(\lambda) & =\frac{1}{2 \pi} \sum_{\tau=-\infty}^{\infty} R_{k}(\tau T+j-k) e^{-i \lambda \tau} \\
& =\frac{1}{2 \pi}\left[\sum_{\tau=-\infty}^{0} R_{k}(\tau T+j-k) e^{-i \lambda \tau}+\sum_{\tau=1}^{\infty} R_{k}(\tau T+j-k) e^{-i \lambda \tau}\right] \\
& =\frac{1}{2 \pi} \exp \left(-\frac{i \lambda(k-j)}{T}\right)\left[A_{k}^{k-j}\left(e^{i \lambda / T}\right)+A_{j}^{T+j-k}\left(e^{-i \lambda / T}\right)\right],
\end{aligned}
$$

for $j>k$,

$$
h_{j k}(\lambda)=\overline{h_{k j}(\lambda)}=\frac{1}{2 \pi} \exp \left(-\frac{i \lambda(k-j)}{T}\right)\left[A_{j}^{j-k}\left(e^{-i \lambda / T}\right)+A_{k}^{T+k-j}\left(e^{i \lambda / T}\right)\right] .
$$

Using (4.8) and (4.9), we arrive at (4.5).

The following theorem gives the spectral density matrix $\mathbf{f}(\lambda)$ in terms of the partitions of PCGF $\left\{A_{l}(x), l=0, \ldots, T-1\right\}$, which are completely determined in Theorem 3.9.

Theorem 4.2. The spectral density of a PCAR $(p)$ process is a Hermitian nonnegative definite $T \times T$ matrix of functions

$$
\mathbf{f}(\lambda)=\left[f_{j k}(\lambda)\right]_{j, k=0,1, \ldots, T-1}, \quad 0<\lambda \leq 2 \pi,
$$


14 Covariance generating functions and spectral densities of PCAR

where

$$
f_{j k}(\lambda)=\frac{1}{2 \pi T^{2}} \sum_{\nu=0}^{T-1} \sum_{l=0}^{T-1} \exp \left(-\frac{2 \pi i(v k-l j)}{T}\right)\left[A_{v}^{\langle v-l\rangle-J_{\langle\nu-l\rangle}}\left(e^{i \lambda / T}\right)+A_{l}^{\langle l-v\rangle}\left(e^{-i \lambda / T}\right)\right] .
$$

Proof. It was proved by Gladyshev [2] that

$$
\mathbf{f}(\lambda)=\frac{1}{T} \mathbf{V}(\lambda) \mathbf{h}(\lambda) \mathbf{V}^{-1}(\lambda)
$$

where $\mathbf{f}(\lambda)$ is the spectral density matrix of the $\left\{X_{t}\right\}$, and $\mathbf{V}(\lambda)$ is a unitary matrix depending on $\lambda$ with elements

$$
U_{j k}(\lambda)=T^{-1 / 2} \exp \left(\frac{2 \pi i j k-i k \lambda}{T}\right)
$$

Note that $\mathbf{V}^{-1}(\lambda)=\mathbf{V}^{*}(\lambda)$, therefore

$$
U_{j k}^{-1}(\lambda)=\overline{U_{j k}(\lambda)}=T^{-1 / 2} \exp \left(\frac{i j \lambda-2 \pi i j k}{T}\right) .
$$

Consequently,

$$
\begin{aligned}
f_{j k}(\lambda)= & \frac{1}{T} \sum_{v=0}^{T-1} \sum_{l=0}^{T-1} U_{j l}(\lambda) h_{l v}(\lambda) U_{v k}^{-1}(\lambda) \\
= & \frac{1}{2 \pi T^{2}} \sum_{v=0}^{T-1} \sum_{l=0}^{T-1} \exp \left(\frac{2 \pi i j l-i l \lambda}{T}\right) \exp \left(-\frac{i \lambda(v-l)}{T}\right) \exp \left(\frac{i v \lambda-2 \pi i v k}{T}\right) \\
& \times\left[A_{v}^{\langle v-l\rangle-J_{\langle v-l\rangle}}\left(e^{i \lambda / T}\right)+A_{l}^{\langle l-v\rangle}\left(e^{-i \lambda / T}\right)\right] \\
= & \frac{1}{2 \pi T^{2}} \sum_{v=0}^{T-1} \sum_{l=0}^{T-1} \exp \left(\frac{2 \pi i(j l-v k)}{T}\right)\left[A_{v}^{\left.\langle v-l\rangle-J_{\langle v-l\rangle}\left(e^{i \lambda / T}\right)+A_{l}^{\langle l-v\rangle}\left(e^{-i \lambda / T}\right)\right] .}\right.
\end{aligned}
$$

In the following, we derive the spectral density matrix of PCAR(1), using (4.11). 
Example 4.3. Let $p=1$, that is, PCAR(1) in Example 3.5. In this case, for any $r=0,1, \ldots$, $T-1,(3.40)$ reduces to

$$
\sum_{l=0}^{1} c_{l}(T-m) x^{l} A_{T-(m+l)}^{\lfloor j+1-l\rfloor_{0}}(x)=e_{j, T-m}(x), \quad m=0,1, \ldots, T-1, j=\lfloor T-m+r\rfloor_{0},
$$

where

$$
e_{j, T-m}(x)= \begin{cases}a_{T-(r+1)}(0), & m=r+1 \\ 0, & \text { otherwise }\end{cases}
$$

Also note that $A_{T-m}^{\left\lfloor\lfloor T-m+r\rfloor_{0}+1\right\rfloor_{0}}(x)=A_{T-m}^{\lfloor T-m+r+1\rfloor_{0}}(x)$ reduces to

$$
A_{T-m}^{\lfloor T-m+r+1\rfloor_{0}}(x)= \begin{cases}A_{T-m}^{T-m+r+1}(x), & -(T-2) \leq-m+r+1 \leq 0, \\ A_{T-m}^{-m+r+1}(x), & 0 \leq-m+r+1 \leq T-1, \\ A_{0}^{0}(x), & -m+r+1=T,\end{cases}
$$

for any $r=0, \ldots, T-1$. Thus the solution of $(4.16)$ is

$$
\begin{aligned}
A_{T-m}^{\lfloor T-m+r+1\rfloor_{0}}(x) & \\
= & \frac{1}{1-\left(\prod_{z=1}^{T} c(z)\right) x^{T}} \\
& \quad \times \begin{cases}c(T-r) \cdots c(T) c(1) \cdots c(T-m) x^{T-r+1-m} a_{T-(r+1)}(0), & -(T-2) \leq-m+r+1 \leq 0, \\
c(T-r) \cdots c(T-m) x^{r+1-m} a_{T-(r+1)}(0), & 0 \leq-m+r+1 \leq T-1, \\
a_{T-(r+1)}(0), & -m+r+1=T .\end{cases}
\end{aligned}
$$

Therefore,

$$
A_{l}^{j}(x)=\frac{1}{1-\tilde{g}(T-1) x^{T}} \begin{cases}C_{l, T+l-j} R_{T+l-j}(0) \tilde{g}(T-1) x^{j}, & l<j, \\ C_{l, l-j} R_{l-j}(0) x^{j}, & l \geq j,\end{cases}
$$

where $\tilde{g}(l)$ is mentioned in Example 3.5 and $C_{j k}=\tilde{g}(j-1) / \tilde{g}(k-1)$. 
16 Covariance generating functions and spectral densities of PCAR

Now, using (4.11), for $j, k=0, \ldots, T-1$, we have

$$
\begin{aligned}
& f_{j k}(\lambda)=\frac{1}{2 \pi T^{2}}\left\{\sum_{v=0}^{T-1} \exp \left(-\frac{2 \pi i v(k-j)}{T}\right)\left[A_{v}^{T}\left(e^{-i \lambda / T}\right)+A_{v}^{0}\left(e^{i \lambda / T}\right)\right]\right. \\
& +\sum_{v=0}^{T-1} \sum_{l=v+1}^{T-1} \exp \left(\frac{2 \pi i(j l-v k)}{T}\right)\left[A_{l}^{l-v}\left(e^{-i \lambda / T}\right)+A_{v}^{T+v-l}\left(e^{i \lambda / T}\right)\right] \\
& \left.+\sum_{v=0}^{T-1} \sum_{l=0}^{v-1} \exp \left(\frac{2 \pi i(j l-v k)}{T}\right)\left[A_{l}^{T+l-v}\left(e^{-i \lambda / T}\right)+A_{v}^{v-l}\left(e^{i \lambda / T}\right)\right]\right\} \\
& =\frac{1}{2 \pi T^{2}}\left\{\sum_{v=0}^{T-1} \exp \left(-\frac{2 \pi i v(k-j)}{T}\right)\left[A_{v}^{T}\left(e^{-i \lambda / T}\right)+A_{v}^{0}\left(e^{i \lambda / T}\right)\right]\right. \\
& +\sum_{v=0}^{T-1} \sum_{l=v+1}^{T-1}\left[\operatorname { e x p } ( \frac { 2 \pi i ( j l - v k ) } { T } ) \left(A_{l}^{l-v}\left(e^{-i \lambda / T}\right)\right.\right. \\
& \left.+A_{v}^{T+v-l}\left(e^{i \lambda / T}\right)\right)+\exp \left(\frac{2 \pi i(j v-l k)}{T}\right)\left(A_{l}^{l-v}\left(e^{i \lambda / T}\right)\right. \\
& \left.\left.\left.+A_{v}^{T+v-l}\left(e^{-i \lambda / T}\right)\right)\right]\right\} \\
& =\frac{1}{2 \pi T^{2}\left|1-\tilde{g}(T-1) e^{i \lambda}\right|^{2}} \\
& \times\left\{\left(1-\tilde{g}^{2}(T-1)\right) \sum_{v=0}^{T-1} \exp \left(-\frac{2 \pi i v(k-j)}{T}\right) R_{v}(0)\right. \\
& +\sum_{v=0}^{T-1} \sum_{l=v+1}^{T-1} \\
& \times\left[\tilde{g}(T-1)\left(C_{v, l} R_{l}(0)-C_{l, v} R_{v}(0)\right)\right. \\
& \times\left(\exp \left(\frac{2 \pi i(j l-v k)+i(v-l+T) \lambda}{T}\right)\right. \\
& \left.+\exp \left(\frac{2 \pi i(l k-j v)-i(v-l+T) \lambda}{T}\right)\right) \\
& +\left(C_{l, v} R_{v}(0)-\widetilde{g}^{2}(T-1) C_{v, l} R_{l}(0)\right) \\
& \left.\left.\times\left(\exp \left(\frac{2 \pi i(j l-v k)+i(v-l) \lambda}{T}\right)+\exp \left(\frac{2 \pi i(l k-j v)-i(v-l) \lambda}{T}\right)\right)\right]\right\},
\end{aligned}
$$

which is obtained in [6]. Note that $\left\{R_{i}(0), i=0, \ldots, T-1\right\}$ are completely specified in Example 3.5. 


\section{Acknowledgments}

The authors are thankful to the referee for providing constructive comments. This research was supported by the Shiraz University Research Council.

\section{References}

[1] P. J. Brockwell and R. A. Davis, Time Series: Theory and Methods, Springer Series in Statistics, Springer, New York, 1991.

[2] E. G. Gladyshev, Periodically correlated random sequences, Soviet Mathematics Doklady 2 (1961), 385-388.

[3] R. P. Grimaldi, Discrete and Combinatorial Mathematics, 3rd ed., Addison-Wesley, Massachusetts, 1994.

[4] A. G. Miamee, Periodically correlated processes and their stationary dilations, SIAM Journal on Applied Mathematics 50 (1990), no. 4, 1194-1199.

[5] A. G. Miamee and H. Salehi, On the prediction of periodically correlated stochastic processes, Multivariate Analysis, V (Proc. Fifth Internat. Sympos., Univ. Pittsburgh, Pittsburgh, Pa, 1978) (P. R. Krishnaiah, ed.), North-Holland, Amsterdam, 1980, pp. 167-179.

[6] A. R. Nematollahi and A. R. Soltani, Discrete time periodically correlated Markov processes, Probability and Mathematical Statistics 20 (2000), no. 1, 127-140.

[7] M. Pourahmadi and H. Salehi, On subordination and linear transformation of harmonizable and periodically correlated processes, Probability Theory on Vector Spaces, III (Lublin, 1983), Lecture Notes in Math., vol. 1080, Springer, Berlin, 1984, pp. 195-213.

[8] H. Sakai, On the spectral density matrix of a periodic ARMA process, Journal of Time Series Analysis 12 (1991), no. 1, 73-82.

[9] Q. Shao and R. B. Lund, Computation and characterization of autocorrelations and partial autocorrelations in periodic ARMA models, Journal of Time Series Analysis 25 (2004), no. 3, 359-372.

[10] A. R. Soltani and A. Parvardeh, Simple random measures and simple processes, Theory of Probability and Its Applications 50 (2005), no. 3, 533-548.

Z. Shishebor: Department of Statistics, College of Science, Shiraz University, Shiraz 71454, Iran E-mail address: sheshebor@susc.ac.ir

A. R. Nematollahi: Department of Statistics, College of Science, Shiraz University, Shiraz 71454, Iran E-mail address: nematollahi@susc.ac.ir

A. R. Soltani: Department of Statistics and Operations Research, College of Science, Kuwait University, P.O. Box 5969, Safat 13060, Kuwait

E-mail address: soltani@kuc01.kuniv.edu.kw

Current address: Department of Statistics, College of Science, Shiraz University, Shiraz 71454, Iran 


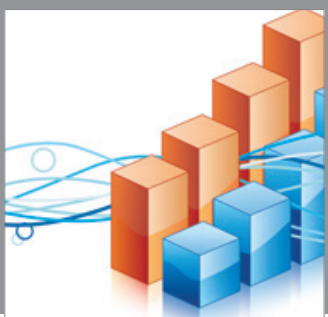

Advances in

Operations Research

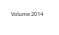

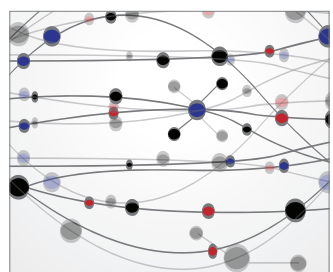

\section{The Scientific} World Journal
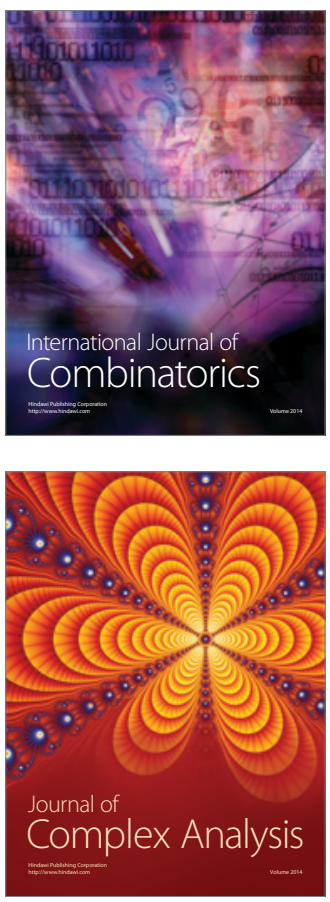

International Journal of

Mathematics and

Mathematical

Sciences
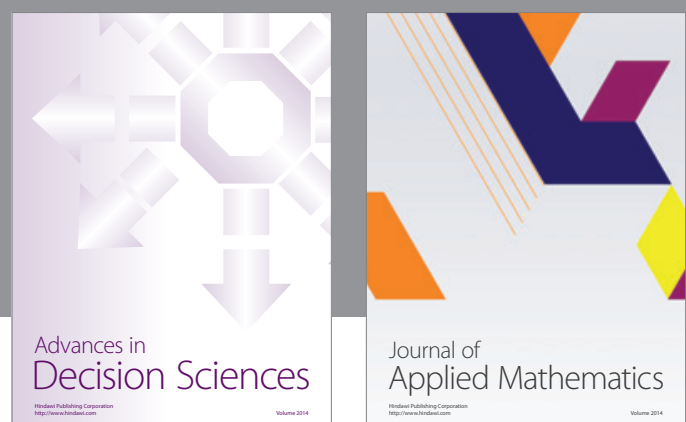

Journal of

Applied Mathematics
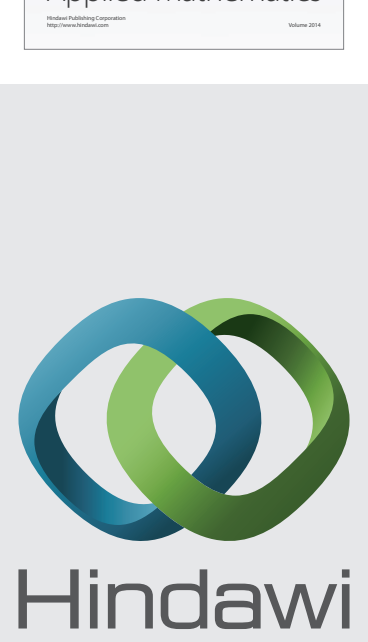

Submit your manuscripts at http://www.hindawi.com
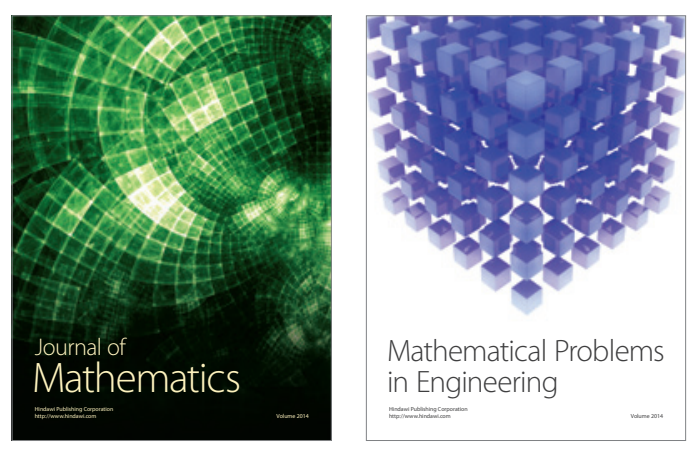

Mathematical Problems in Engineering
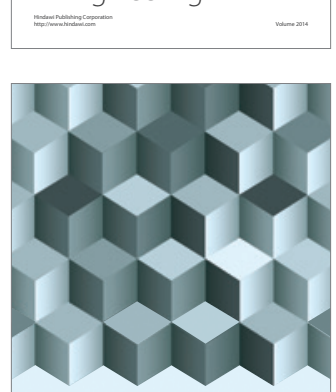

Journal of

Function Spaces
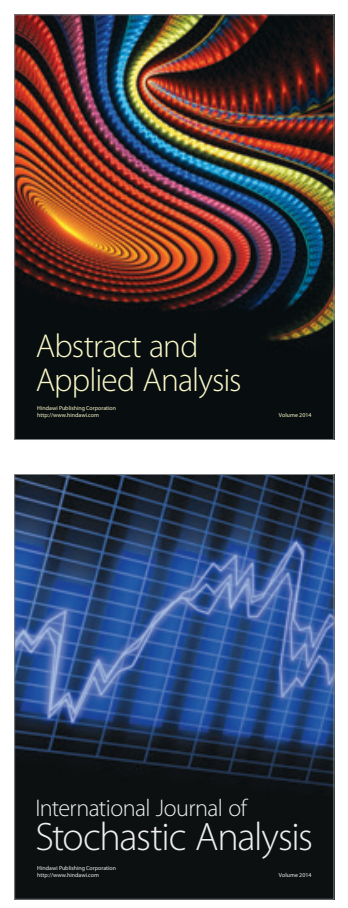

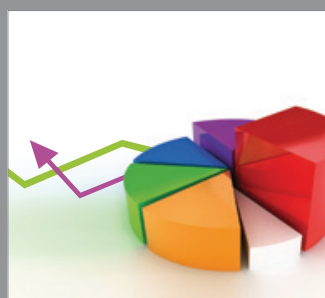

ournal of

Probability and Statistics

Promensencen
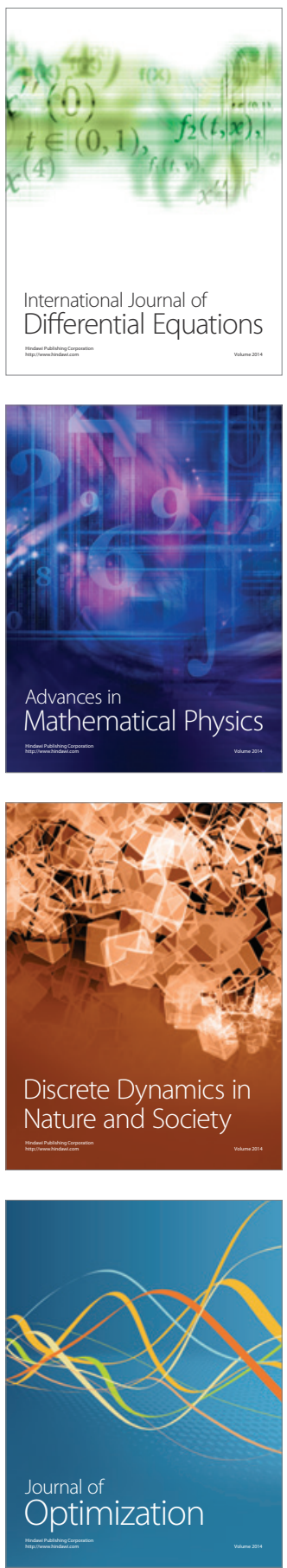